\title{
Factores que influyen en el reingreso hospitalario del adulto mayor: resultados de un plan piloto
}

\section{factors that influence in the hospital reign of the elderly adult: results of a pilot plan}

Jessica Daniela Altamirano Llinin. ${ }^{1}$ \& Milagros Orozco Reyes. ${ }^{2}$

Recibido: 04-03-2019 / Revisado: 15-04-2019 /Aceptado: 24-05-2019/ Publicado: 05-06-2019

\begin{abstract}
.
DOI: https://doi.org/10.33262/cienciadigital.v3i2.6.568

The lack of knowledge of self-care in the elderly is one of the main factors of a hospital readmission, the research determining the factors that influence the hospital readiness of the elderly. For this study, quantitative research of transverse origin was implemented, descriptive research was used. The population was 120 patients being these adults, applying a pilot plan of 20 patients included in the year 2018, as an instrument was used an observation sheet that consists of 4 factors which were validated by health experts checking in this way its validity and coherence. In the analysis of the observation card, the lack of self-care knowledge of the older adult stood out $58 \%$, diabetes mellitus I as the most frequent disease in the rehospitalization with $48 \%$, followed by a severe clinical state $27 \%$ and finally as a greater diagnosis of re-enter the recurrence of the disease with $44 \%$, concluding that the lack of knowledge about a guide to self-care in older adults gave as a higher percentage at the time of hospital readmission.
\end{abstract}

Key Words: Older adult, hospital re-entry, self-care.

\section{Resumen.}

La falta de conocimiento del autocuidado en el adulto mayor es uno de los factores principales de un reingreso hospitalario, la investigación determinar los factores que influyen en el reingreso hospitalario del adulto mayor. Para este estudio se implementó la investigación cuantitativa, de origen transversal, se empleó la investigación

1 Universidad Estatal de Milagro, Milagro, Ecuador, jaltamirano1987@hotmail.com

2 Universidad Estatal De Milagro. Milagro, Ecuador, m.orozco.mo@gmail.com 
descriptiva. La población fue de 120 pacientes siendo estos adultos mayores, aplicando un plan piloto de 20 pacientes comprendidos en el año 2018, como instrumento se utilizó una ficha de observación que consta de 4 factores los cuales fueron validados por expertos en la salud comprobando de esta manera su validez y coherencia.

En el análisis de la ficha de observación sobresalió la falta de conocimiento de autocuidado del adulto mayor 58\%, la diabetes mellitus I como enfermedad más frecuente en el reingreso con el $48 \%$, seguido de estado clínico grave $27 \%$ y finalmente como mayor diagnóstico de reingreso la recurrencia de la enfermedad con el $44 \%$, concluyendo que la falta de conocimiento sobre una guía de autocuidado en adulto mayor dio como mayor porcentaje al momento de darse un reingreso hospitalario.

Palabras claves: Adulto mayor, reingreso hospitalario, autocuidado

\section{Introducción.}

La trayectoria de vida de los hombres y las mujeres los colocan con una marcada diferencia en la salud en edades de la vejez, que reflejan los estilos de vida que adoptaron a lo largo de su existencia. Debido a su rol social como proveedor económico de la familia, así como a su actividad más intensa en el mercado laboral, los hombres en general pueden estar sujetos a mayores presiones físicas y mentales que ponen en riesgo su organismo. (Salgado de Snyder \& Wong, 2007)

Las personas de 60 a 74 años según la OMS son consideradas de edad avanzada, de 75 a 90 viejas o ancianas, y las que sobrepasan los 90 se les designa grandes viejos o grandes longevos. A todo individuo mayor de 60 años se le llamará de forma indistinta persona de la tercera edad o adulto mayor. (Dionne Espinosa, 2010)

El mayor problema que se presenta en esta población radica en aspectos de salud, son personas vulnerables a sufrir constantemente cualquier tipo de enfermedad.

Los cambios que componen e influyen el envejecimiento son complicados. En el plano biológico, el envejecimiento está asociado con la acumulación de una gran variedad de daños celulares y moleculares. Al pasar del tiempo estos daños reducen gradualmente las reservas fisiológicas, aumentan el riesgo de muchas enfermedades y disminuyen en general la capacidad del individuo. A la larga, sobreviene la muerte. (Organización Mundial de la Salud, 2015)

El adulto mayor, tienen mayores riesgos de contraer enfermedades degenerativas del organismo, siendo una de ellas y la más frecuente la diabetes mellitus, ya que los niveles de glucemia en ayunas se incrementa con la edad avanzada, por ende una resistencia a la insulina 
y disminución en la secreción de la misma en los ancianos. (Bados, Carmona, Coppola, \& De la Cruz, 2016)

Un reingreso hospitalario es aquel que se produce luego de un ingreso previo, es adecuado el término ingreso repetido para definir la situación general. Es aquel producido por la misma patología en un tiempo determinado. (Malagón, Pontón, \& Galán , 2008)

Los reingresos hospitalarios son un significativo reflejo de la complejidad tanto de las relaciones entre los distintos niveles asistenciales como de la atención a personas con edad avanzada. Un ingreso hospitalario en estas personas es un factor de riesgo que puede desencadenar un proceso de declive en el estado funcional y una pérdida de calidad de vida. (Rios Palacio, 2015)

El autocuidado refuerza la participación activa de las personas en el cuidado de su salud, responsables de decisiones que condicionan su situación actual, manejando aspectos que se relacionan con la motivación y cambio de comportamiento, donde la educación para la salud es la principal herramienta de trabajo. (Sanhueza Parra, Castro Salas, \& Merino Escobar, 2012)

Es por eso la importancia de este estudio para poder disminuir el reingreso hospitalario del adulto mayor, y la mejor manera es dándoles a conocer sobre su autocuidado.

\section{Metodologia.}

Para este estudio se utilizó el método analítico - sintético, ya que sirvió para indagar de forma individual cada uno de ellos problemas que presenta el adulto mayor al momento de su reingreso hospitalario, para luego integrar estos problemas y observar y observar como influyó en mejorar la calidad de vida del adulto mayor para evitar así una recaída en su salud que conlleve a un reingreso hospitalario.

Como método empírico se utilizó el método de la observación, un método fundamental que fue empleado durante todo el trabajo investigativo, observando de esta manera cada situación que se presentó principalmente en el momento de aplicar la ficha de recolección de datos al adulto mayor que tuvo un reingreso hospitalario en el área de medicina interna del Hospital General Milagro.

Se consideró como técnica e instrumentos para este estudio, la ficha de recolección de datos. La ficha estuvo integrada por 4 factores, logrando obtener información valiosa que ayudaron a comprobar los objetivos de esta investigación, la ficha de recolección de datos se aplicó al adulto mayor que tuvo un reingreso hospitalario en el área de medicina interna del Hospital General Milagro. La ficha se aplicó a 120 personas. Se aplicó un plan piloto a 20 pacientes comprendido en el periodo 2018. 
La ficha de recolección de datos está dirigida en relación a cada objetivo de la investigación. $\mathrm{Su}$ objetivo principal radica en determinar los factores que influyen en el reingreso hospitalario del adulto mayor en el servicio de medicina interna del Hospital General Milagro, en el cual encontramos en la ficha el ítem factores que influyen en el reingreso; (descuido familiar, mal manejo terapéutico y falta de conocimiento del autocuidado).

Para obtener información para este estudio nos referimos al área de medicina interna del Hospital General Milagro observando el historial clínico d cada paciente.

La ficha de observación contiene los siguientes puntos con sus respectivas respuestas objetivas; Los factores que influyen en el reingreso hospitalario (descuido familiar, mal manejo terapéutico, falta de conocimiento autocuidado), enfermedad más frecuente que presenta el adulto mayor en un reingreso hospitalario (hipertensión, diabetes mellitus I, diabetes mellitus II, gastroenteritis, evento cerebro vascular, otro), el estado clínico al momento del reingreso hospitalario (muy grave, grave, medianamente grave, poco grave, nada grave) y como último punto analizamos el diagnóstico de reingreso del adulto mayor (recurrencia de la enfermedad, adherencia al tratamiento, nuevas enfermedades, reingreso múltiple, otro motivo).

La herramienta que se utilizó para el análisis estadístico fue la de IMB SPSS Statistcs Versión 22, por medio del cual nos permitió ingresar los datos para luego proceder a la tabulación e ilustración de las tablas estadísticas mostrando la frecuencia, porcentaje válido y porcentaje acumulado.

\section{Resultados.}

En el análisis comparativo de los cuadros estadísticos realizado al adulto mayor en el área de medicina interna del Hospital General Milagro, nos da a conocer que el 58\% del adulto mayor tuvo un reingreso hospitalario por falta de conocimiento sobre su autocuidado.

En el siguiente análisis se determina los factores que influyen en el reingreso hospitalario del adulto mayor, donde se analizó 20 casos.

El factor más importante que influye en el reingreso hospitalario del adulto mayor es la falta de conocimiento de autocuidado que corresponde al $60 \%$ de los 20 pacientes seleccionados para el plan piloto, el $25 \%$ corresponde a mal manejo terapéutico y el $15 \%$ a descuido familiar como podemos observar en la tabla $\mathrm{N}^{\circ} 1$. 
Tabla $\mathbf{N}^{\circ}$ 1. Factores que influyen en el reingreso hospitalario del adulto mayor

Factores que influyen en el reingreso hospitalario del adulto mayor.

\begin{tabular}{llcccc}
\hline & Frecuencia & $\begin{array}{l}\text { Porcentaje } \\
\text { \% }\end{array}$ & $\begin{array}{l}\text { Porcentaje } \\
\text { válido \% }\end{array}$ & $\begin{array}{c}\text { Porcentaje } \\
\text { acumulado }\end{array}$ \\
$\begin{array}{l}\text { Descuido familiar } \\
\text { Mal manejo }\end{array}$ & 3 & 15 & 15 & 15 \\
$\begin{array}{l}\text { Válido } \\
\text { terapéutico }\end{array}$ & 5 & 25 & 25 & 40 \\
$\begin{array}{l}\text { Falta de } \\
\text { conocimiento } \\
\text { autocuidado }\end{array}$ & 12 & 60 & 60 & 100 \\
Total & $\mathbf{2 0}$ & $\mathbf{1 0 0}$ & $\mathbf{1 0 0}$ & \\
\hline
\end{tabular}

Elaborado por: Grupo de investigación

En este análisis de estudio, se pudo verificar la enfermedad más frecuente al momento de darse el reingreso hospitalario por parte del adulto mayor, en el cual 8 de los 20 pacientes sufren de diabetes mellitus I el cual forma parte del $40 \%$ de los pacientes, en segundo lugar verificamos a la hipertensión con el $20 \%$, seguido de la diabetes mellitus II y con un mismo porcentaje del $10 \%$ la gastroenteritis y evento cerebrovascular, tal como se detalla en la tabla $\mathrm{N}^{\circ} 2$.

Tabla $\mathbf{N}^{\circ}$ 2. Enfermedad más frecuente en el reingreso del adulto mayor

Enfermedad más frecuente en el reingreso del adulto mayor

\begin{tabular}{llcccc}
\hline & Frecuencia & Porcentaje & $\begin{array}{c}\text { Porcentaje } \\
\text { válido }\end{array}$ & $\begin{array}{c}\text { Porcentaje } \\
\text { acumulado }\end{array}$ \\
\cline { 3 - 4 } & Hipertensión & 4 & 20 & 20 & 20 \\
& Diabetes mellitus I & 8 & 40 & 40 & 60 \\
& Diabetes mellitus II & 3 & 15 & 15 & 75 \\
& Gastroenteritis & 2 & 10 & 10 & 85 \\
& Evento cerebro & 2 & 10 & 10 & 95 \\
& vascular & 1 & 5 & 5 & 100 \\
& Otro & $\mathbf{2 0}$ & $\mathbf{1 0 0}$ & $\mathbf{1 0 0}$ & \\
\hline
\end{tabular}

Elaborado por: Grupo de investigación 
Para el siguiente análisis se revisó la historia clínica de cada paciente para analizar el estado clínico en que llegan al centro de salud al momento de darse el reingreso hospitalario, primero se determinó que el $55 \%$ de los pacientes llegan en un estado clínico poco grave, seguido del $30 \%$ grave y el $15 \%$ muy grave. Así lo detallamos en la tabla $\mathrm{N}^{\circ} 3$.

Tabla $\mathbf{N}^{\circ}$ 3. Estado clínico del reingreso del adulto mayor

Estado clínico al momento del reingreso del adulto mayor

\begin{tabular}{clcccc}
\hline & Frecuencia & Porcentaje & $\begin{array}{l}\text { Porcentaje } \\
\text { válido }\end{array}$ & $\begin{array}{l}\text { Porcentaje } \\
\text { acumulado }\end{array}$ \\
Válido & $\begin{array}{l}\text { Muy } \\
\text { grave }\end{array}$ & 3 & 15 & 15 & 15 \\
& $\begin{array}{l}\text { Grave } \\
\text { Poco }\end{array}$ & 6 & 30 & 30 & 45 \\
& $\begin{array}{l}\text { grave } \\
\text { Total }\end{array}$ & 11 & 55 & 55 & 100 \\
\hline
\end{tabular}

Elaborado por: Grupo de investigación

Con respecto al diagnóstico de reingreso hospitalario del adulto mayor se determinó la recurrencia de la enfermedad con el mayor número de pacientes esto es un $40 \%$, reingreso múltiple con el $25 \%$, seguido de adherencia al tratamiento con un $20 \%$ y finalmente nuevas enfermedades con un $15 \%$, como podemos observar en el detalle de la tabla $\mathrm{N}^{\circ} 4$.

Tabla $\mathbf{N}^{\circ}$ 4. Diagnóstico del reingreso hospitalario del adulto mayor

Diagnóstico de Reingreso Hospitalario en el adulto mayor

\begin{tabular}{|c|c|c|c|c|c|c|}
\hline & & & Frecuencia & Porcentaje & $\begin{array}{l}\text { Porcentaje } \\
\text { válido }\end{array}$ & $\begin{array}{l}\text { Porcentaje } \\
\text { acumulado }\end{array}$ \\
\hline \multirow{5}{*}{ Válido } & $\begin{array}{l}\text { Recurrencia de } \\
\text { enfermedad }\end{array}$ & la & 8 & 40 & 40 & 40 \\
\hline & $\begin{array}{l}\text { Adherencia } \\
\text { tratamiento }\end{array}$ & al & 4 & 20 & 20 & 60 \\
\hline & Nuevas enfermedades & & 3 & 15 & 15 & 75 \\
\hline & Reingreso múltiple & & 5 & 25 & 25 & 100 \\
\hline & Total & & 20 & 100 & 100 & \\
\hline
\end{tabular}

Elaborado por: Grupo de investigación 


\section{Discusión}

En el presente plan piloto realizado a 20 pacientes pudimos verificar que el factor más importante que influye en el reingreso hospitalario es la falta de conocimiento de autocuidado por parte del adulto mayor con un $60 \%$ de los pacientes.

Sin embargo, en la tesis doctoral Factores predictores de reingresos tempranos en pacientes ancianos con multimorbilidad ingresados en una unidad de cuidados intensivos, se muestra como causa principal de un reingreso del adulto mayor enfermedad pulmonar obstructiva crónica, neumonía o insuficiencia cardiaca descompensada. (Sánchez Rodríguez, 2017)

En el artículo Rehospitalización en adultos mayores de un hospital de Lima, Perú; se encontró que la tasa de rehospitalización fue $14 \%$ lo cual se encuentra dentro del rango que presentan países de Latinoamérica $(9-23 \%)$, Norteamérica $(13-23 \%)$, Canadá (13.3\%) y Europa (5 - 14\%). En este estudio se puede concluir que el factor de riesgo más importante para la rehospitalización en adultos mayores es la falta de visitas de control posterior al alta en los pacientes. (Quispe, Ticse, Gálvez, \& Valera, 2013)

Por lo tanto al comparar los resultados de la tesis doctoral y el artículo de rehospitalización, se pudo verificar resultados diferentes ya que en la tesis doctoral el principal factor de un reingreso es causado por una enfermedad pulmonar obstructiva crónica, mientras que en este estudio se constató a la falta de conocimiento del autocuidado del adulto mayor como principal factor por reingreso hospitalario.

\section{Conclusiones.}

- Se determinó en el presente plan piloto que el factor más importante que se da por un reingreso hospitalario en el Hospital General Milagro 2018, fue la falta de conocimiento del autocuidado del adulto mayor.

- Se pudo identificar también que la enfermedad más frecuente en el reingreso hospitalario del adulto mayor fue la Diabetes mellitus I, seguido de la hipertensión.

- Por otro lado se pudo constatar el estado clínico al momento de darse un reingreso hospitalario por parte del adulto mayor, el cual pudimos ver que la mayoría de pacientes ingresan en estado poco grave y no hubo registros de mortalidad debido a un reingreso.

- Se pudo identificar también el diagnóstico de reingreso hospitalario en el adulto mayor al momento de darse el reingreso, el cual predomino la recurrencia a la enfermedad, seguido de reingreso múltiple.

- Por lo tanto, se concluye que el factor más importante al darse un reingreso hospitalario en el adulto mayor fue la falta de conocimiento en el autocuidado del mismo. 


\section{Referencias bibliográficas.}

Bados, F., Carmona, L., Coppola, U., \& De la Cruz, S. (2016). Universidad Peruana Cayetano Heredia. Recuperado el 4 de abril de 2019, de http://repositorio.upch.edu.pe/bitstream/handle/upch/623/Adherencia\%20al\%20r\%C3\% A9gimen\%20terap\%C3\%A9utico\%20de\%20los\%20pacientes\%20adultos\%20mayores\%20c on\%20diabetes\%20mellitus\%20tipo\%20II\%20y\%20frecuencia\%20de\%20ingreso\%20al\%20 servicio\%20de\%20emergencia

Dionne Espinosa, M. (2010). Universidad de las Américas Puebla. Recuperado el 04 de abril de 2019, de http://catarina.udlap.mx/u_dl_a/tales/documentos/lar/dionne_e_mf/capitulo1.pdf

Malagón, G., Pontón, G., \& Galán , R. (2008). Administración Hospitalaria (Tercera Edición ed.). Bogotá, Colombia: Editorial Médica Panamericana.

Organización Mundial de la Salud. (2015). Informe mundial sobre el envejecimiento y la salud. Ediciones de la OMS.

Quispe, T., Ticse, R., Gálvez, M., \& Valera, L. (2013). Rev. Perú Med Exp Salud Pública. Recuperado el 04 de abril de 2019, de http://www.scielo.org.pe/pdf/rins/v30n4/a16v30n4.pdf

Rios Palacio, J. (Octubre de 2015). Universidad Tecnológica de Pereira . Recuperado el 04 de abril de 2019, de http://repositorio.utp.edu.co/dspace/bitstream/handle/11059/5764/6168527R586.pdf;js essionid=F8AA288B459BBFB5E29CD2385E62AD78? sequence $=1$

Salgado de Snyder, N., \& Wong, R. (2007). Género y pobreza: Determinantes de la salud en la vejez . Medigraphic Artemisa en línea .

Sánchez Rodríguez, J. L. (2017). Universidad Complutense de Madrid. Recuperado el 04 de abril de 2019, de https://eprints.ucm.es/44529/1/T39238.pdf

Sanhueza Parra, M., Castro Salas, M., \& Merino Escobar, J. (2012). Optimizando la funcionalidad del adulto mayor a través de una estrategia de autocuidado.

\section{UL Ciencia}




\section{PARA CITAR EL ARTÍCULO INDEXADO.}

Altamirano Llinin, J., \& Orozco Reyes, M. (2019). Factores que influyen en el reingreso hospitalario del adulto mayor: resultados de un plan piloto. Ciencia Digital, 3(2.6), 299-307. https://doi.org/10.33262/cienciadigital.v3i2.6.568

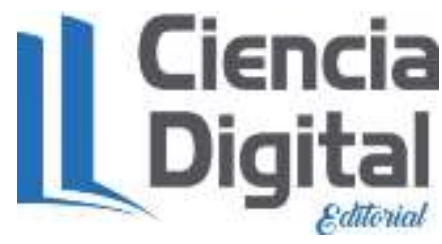

El artículo que se publica es de exclusiva responsabilidad de los autores y no necesariamente reflejan el pensamiento de la Revista Ciencia Digital.

El artículo queda en propiedad de la revista y, por tanto, su publicación parcial y/o total en otro medio tiene que ser autorizado por el director de la Revista Ciencia Digital.
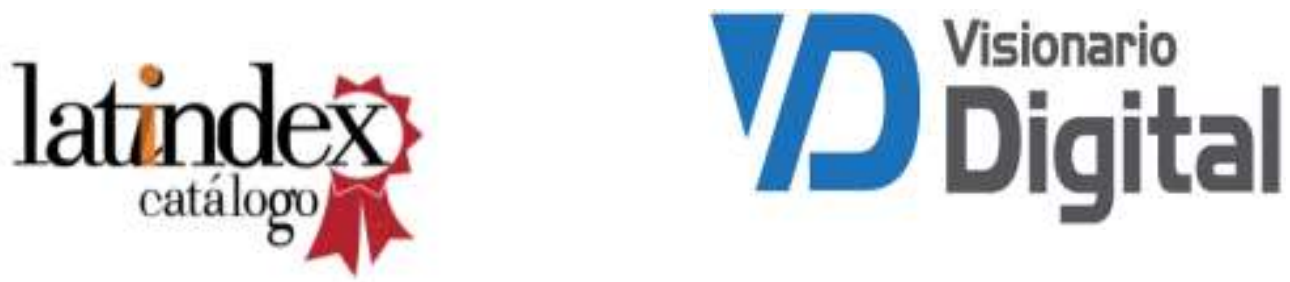